\title{
Monitoring of Building Construction by 4D Change Detection Using Multi-temporal SAR Images
}

\author{
C. H. Yang ${ }^{\mathrm{a}, *}$, Y. Pang ${ }^{\mathrm{b}}, \mathrm{U}$. Soergel ${ }^{\mathrm{a}}$

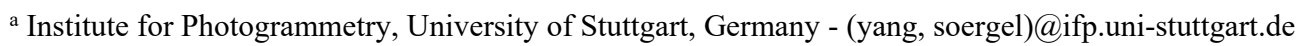 \\ b Institute for Photogrammetry, University of Stuttgart, Germany - pangye.rs@gmail.com
}

ISPRS Hannover Workshop 2017

KEY WORDS: Persistent Scatterer Interferometry (PSI), Synthetic Aperture Radar (SAR), Change Detection, Urban Monitoring, Time Series Analysis, Object-based Analysis

\begin{abstract}
:
Monitoring urban changes is important for city management, urban planning, updating of cadastral map, etc. In contrast to conventional field surveys, which are usually expensive and slow, remote sensing techniques are fast and cost-effective alternatives. Spaceborne synthetic aperture radar (SAR) sensors provide radar images captured rapidly over vast areas at fine spatiotemporal resolution. In addition, the active microwave sensors are capable of day-and-night vision and independent of weather conditions. These advantages make multi-temporal SAR images suitable for scene monitoring. Persistent scatterer interferometry (PSI) detects and analyses PS points, which are characterized by strong, stable, and coherent radar signals throughout a SAR image sequence and can be regarded as substructures of buildings in built-up cities. Attributes of PS points, for example, deformation velocities, are derived and used for further analysis. Based on PSI, a 4D change detection technique has been developed to detect disappearance and emergence of PS points (3D) at specific times (1D). In this paper, we apply this 4D technique to the centre of Berlin, Germany, to investigate its feasibility and application for construction monitoring. The aims of the three case studies are to monitor construction progress, business districts, and single buildings, respectively. The disappearing and emerging substructures of the buildings are successfully recognized along with their occurrence times. The changed substructures are then clustered into single construction segments based on DBSCAN clustering and $\alpha$-shape outlining for object-based analysis. Compared with the ground truth, these spatiotemporal results have proven able to provide more detailed information for construction monitoring.
\end{abstract}

\section{INTRODUCTION}

Human activities, such as population growth, economic globalization, urban extension, and natural disasters have led to frequent urban changes, which mostly regard building construction. Monitoring such changes is important for city management, urban planning, updating of cadastral map, environmental monitoring, disaster assessment, etc. (Gamba, 2013; Marin et al., 2015). In contrast to conventional field surveys, which are usually expensive and slow, remote sensing techniques are fast and cost-effective alternatives. Spaceborne synthetic aperture radar (SAR) sensors provide radar images captured rapidly over vast areas at fine spatiotemporal resolution. For example, a single TerraSAR-X (TSX) image acquired in Stripmap mode covers a standard scene size of $50 \mathrm{~km} \times 30 \mathrm{~km}$, which can be repeatedly scanned every 11 days, with spatial resolution of $3 \mathrm{~m}$. The spatial resolution can be increased up to $20 \mathrm{~cm}$ when Staring Spotlight mode is operated (Mittermayer et al., 2014). In addition, the active microwave sensors are capable of day-and-night vision and independent of weather conditions. The advantages mentioned above make SAR suitable for monitoring tasks.

Time series analysis based on SAR images is widely used for urban monitoring. Persistent scatterer interferometry (PSI) (Crosetto et al., 2016; Ferretti et al., 2000, 2001, 2011; Hooper et al., 2004; Kampes, 2006) is a technique to detect and analyse PS points, which are characterized by strong, stable, and coherent radar signals throughout a SAR image sequence and can be regarded as substructures of buildings in built-up cities.
Attributes of PS points, including line-of-sight velocities (mm/year level), topography heights, and geographic positions, can be derived and used for monitoring of structural deformation and 3D building modelling. A prerequisite of forming PS points is that their signals must maintain coherence throughout a time series of SAR images. For example, to avoid coherence loss, buildings covered with PS-like substructures are modelled to be steady and free of any big changes during the entire acquisition period of the SAR image stack. In contrast, if the substructures disappear or emerge arbitrarily due to construction, the corresponding temporary PS points are discarded at the initial screening of temporally stable scatterers in a standard PSI processing.

Certain approaches (Ansari et al., 2014; Brcic and Adam, 2013; Ferretti et al., 2003; Novali et al., 2004) are dedicated to retrieving temporary PS points, which exist only in a portion of a SAR image sequence due to big changes. Our previous work proposed a 4D change detection technique to recognize two types of temporary PS points, which are called disappearing big change (DBC) and emerging big change (EBC) points, along with their occurrence times (Yang et al., 2016b). This technique has been validated by simulated and real data tests.

In this paper, we apply our further developed method to enable construction monitoring in more detail. The spatiotemporal change detection result reveals a global picture of $\mathrm{DBC}$ and $\mathrm{EBC}$ points distributed over a built-up area $(5 \mathrm{~km} \times 5 \mathrm{~km})$. Each DBC and $\mathrm{EBC}$ point indicate disappearance and emergence,

* Corresponding author 
respectively, of a substructure on a building at a specific time. We focus on single buildings to explore their construction progresses in detail, e.g., completion times of main structures, supplemented substructures, and storeys. In order to move towards object analysis, we cluster spatially correlated DBC or EBC points of similar temporal evolution pattern into segments.

This paper is organized as follows. We describe PSI, our change detection approach, and clustering scheme in Sections 2, 3, and 4 , respectively. Section 5 discusses the experiments in depth based on pixel-based analysis. The object-based analysis is then followed in Section 6. Finally, we summarize the conclusions and future works in Section 7.

\section{PERSISTENT SCATTERER INTERFEROMETRY}

Input data are a time series of $N$ spatially overlapping complex SAR images acquired from the same orbit. So-called slave images are precisely co-registered to a master image, which is selected under small baseline constraint (Berardino et al., 2002; Lanari et al., 2004). Then, $N$-1 interferograms between the master and all of the slave images are computed. The interferometric phases of each pixel are used to estimate its temporal coherence, line-of-sight velocity, and relative topography height via a periodogram search (Ferretti et al., 2001). The temporal coherence serves as a measure of phase stability throughout the SAR images. Finally, pixels with high temporal coherences are selected as PS points. However, DBC and EBC points, if any, are just discarded in standard PSI as they suffer coherence loss during the entire image sequence. To retrieve such big change information, we resort to our approach described in the next section.

\section{4D CHANGE DETECTION TECHNIQUE}

\subsection{Single-break-date Change Detection}

We first illustrate the change detection scheme subject to a single break date that big changes occur before or after. Complete, front, and back SAR image sets are defined from an image sequence for use in this scheme. The complete set consists of all of the images in the sequence. The front and back sets comprise the images taken before and after some arbitrarily chosen break date, respectively. Our aim is to find PS points that exist in the front set but disappear in the back set and vice versa. The PS points that suddenly disappear are termed $\mathrm{DBC}$ points and those emerging in the back set are called EBC points.

The flowchart of single-break-date change detection (Figure 1) is composed of the persistence, disappearance, and emergence scenarios, in which the complete, front, and back sets are mainly involved, respectively. These three image sets are processed by a standard PSI procedure to generate three temporal coherence images. The temporal coherence of a $\mathrm{DBC}$ or $\mathrm{EBC}$ point in the front or back set is supposed to be higher than that in the complete set, which is reduced due to the big change. Based on this assumption, the change indices of a pixel $x$ in the disappearance $\left(C I^{\mathrm{D}}\right)$ and emergence $\left(C I^{\mathrm{E}}\right)$ scenarios are calculated by

$$
\begin{aligned}
& C I^{\mathrm{D}}(x)=\gamma_{\mathrm{T}}^{\mathrm{F}}(x)-\gamma_{\mathrm{T}}^{\mathrm{C}}(x)(1) \\
& {[-1,+1] \in \mathrm{R}} \\
& C I^{\mathrm{E}}(x)=\gamma_{\mathrm{T}}^{\mathrm{B}}(x)-\gamma_{\mathrm{T}}^{\mathrm{C}}(x)(2) \\
& {[-1,+1] \in \mathrm{R}}
\end{aligned}
$$

where $\gamma_{\mathrm{T}}^{\mathrm{C}}, \gamma_{\mathrm{T}}^{\mathrm{F}}$, and $\gamma_{\mathrm{T}}^{\mathrm{B}}$ denote the temporal coherences in the complete, front, and back sets. A pixel is more likely to be a DBC or $\mathrm{EBC}$ point when $C I^{\mathrm{D}}$ or $C I^{\mathrm{E}}$ is closer to 1 , respectively. A change index distribution over DBC or EBC points is modelled to be a right-tailed probability function towards 1 . Then, a statistical-based thresholding is applied to the change indices of the pixels to extract DBC and EBC points. The extracted points are jointly analysed with the PS points, which are selected in the persistence scenario, to reject two types of outliers. First, PS points are discarded if they coincide with either of the other two point labels. Second, a DBC point must not be an EBC point as well and vice versa. Finally, the PS, DBC, and EBC points are combined into a change detection result. However, the accurate times of the big changes are lacking as they are only known to disappear and emerge after and before the break date, respectively.

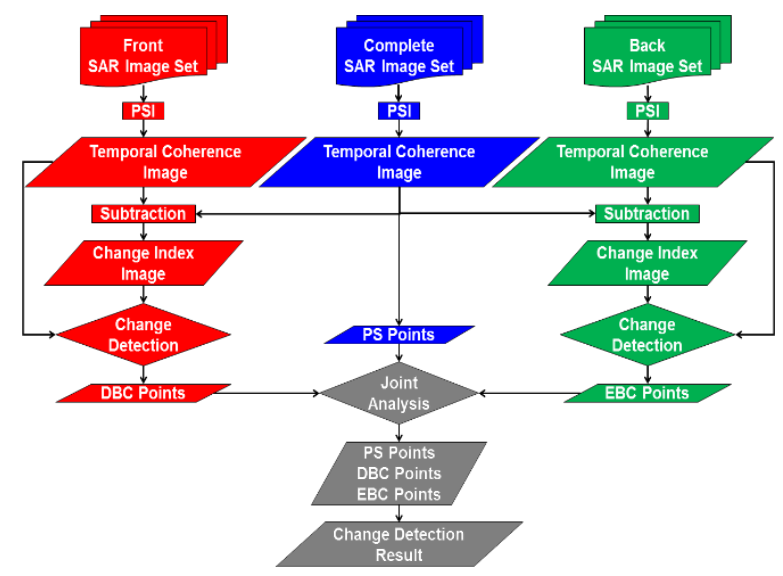

Figure 1. Flowchart of single-break-date change detection. Persistence (blue), disappearance (red), and emergence (green) scenarios are dedicated to extracting PS, DBC, and EBC points, respectively.

\subsection{Multi-break-date Change Detection}

To detect accurate big change times, a set of single-break-date results are jointly analysed in a multi-break-date change detection (Figure 2). For each pixel, two sequences, i.e., change indices and initial point labels (PS, DBC, and, EBC), have been determined thus far spanning a certain temporal period of interest. These two sequences are now analysed in depth. Some of the initial labels may be erroneous due to processing uncertainty. The majority vote is then applied to each label sequence to determine the pixel' label, e.g., a pixel is labelled PS if most of its initial labels are PS. Nevertheless, false labels are still unavoidable but can be removed by an outlier filtering. Three outlier types are described below along with their removal strategies using sliding window operation.

- Homogeneous points are expected to form a single object. For example, PS points are unlikely to appear on a demolished apartment full of DBC points. For this reason, $\mathrm{PS}, \mathrm{DBC}$, or $\mathrm{BC}$ points, which are in the majority in a window, are retained; the other inconsistent points are then deleted.

- An isolated PS, DBC, or EBC point in a window is removed considering that its reliability cannot be inspected by comparing with neighbours.

- A PS point is removed if its velocity is too large or quite different from the velocities of the neighbouring PS points in a window.

Then, the change date of each $\mathrm{DBC}$ or EBC point is estimated from the time-series break dates based on the temporal variation in its change index sequence. The concept is to detect the turning point of a change index sequence, which is modelled to correspond to a disappearance or emergence date. In the end, the 
PS, DBC, and EBC points along with the change dates are fused to illustrate the spatiotemporal changes.

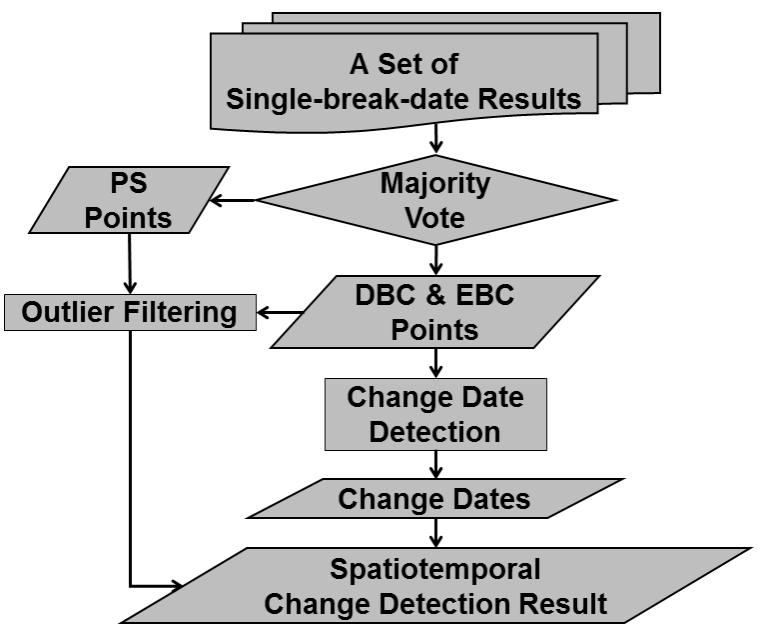

Figure 2. Flowchart of multi-break-date change detection.

\section{CLUSTERING SCHEME}

We use DBSCAN (Ester et al., 1996) to cluster DBC and EBC points into independent segments, which may indicate separate construction events. This clustering algorithm is not subject to a specified number of segments and can be adapted to arbitrary shapes. These two characteristics are suitable to cluster DBC or EBC points as their local amounts and spatial density vary. In initial clustering stage, all points are classified as core, reachable, and noise points. A point is defined as a core point if at least a minimum number of the neighbouring points are reachable from its position within a maximum radius. These neighbouring points are thus called reachable points to the core point. In addition, a point outside the maximum radius is also defined as a reachable point if it can be indirectly connected to the core point via other adjacent core points. By definition, a core point can also be reachable points to other core points. Those points that are neither core nor reachable points are then classified as noise points. Finally, a core point and its reachable points are clustered as one single segment. Finally, each segment's shape is derived by $\alpha-$ shape outlining (Edelsbrunner et al., 1983), which is capable of determining an arbitrary shape of a set of finite points in a plane. The clustered $\mathrm{DBC}$ or EBC points inside each segment are mutually connected by Delaunay triangulation. Those points along the outer edge are then linked to form a closed shape.

\section{DEMONSTRATION OF CONSTRUCTION MONITORING}

This test adopts forty TSX images (Table 1) acquired in High Resolution Spotlight mode from October 27, 2010 to September 4, 2014. PSI requires a minimum amount of images, which coincide with lower limits of front and back image sets. Thirteen break dates were chosen between the $16^{\text {th }}$ and $29^{\text {th }}$ acquisition dates, i.e., we conducted thirteen single-break-date instances that were jointly processed by multi-break-date change detection. All of the images are precisely co-registered and resampled into 5000 $\times 5000$ grid of ground resolution of $1 \mathrm{~m}$, which is able to represent detailed substructures. The study area (Figure 3) covering the city centre in Berlin, Germany, shows many bright clusters of strong signals on buildings that appear to be potential PS points. Three Google Earth (GE) images (ground truth) taken on September 12, 2010, May 20, 2012, and September 5, 2014 reveal that many construction activities took place within the image acquisition period. The spatiotemporal change detection result (Figure 4) reveals the locations and dates of various building erection and demolition events (The examples of a DBC and EBC points are shown in Appendix). We focus the following discussion on highlighted patches 1 to 3 .

\begin{tabular}{|c|c|c|c|}
\hline \multicolumn{4}{|c|}{ Acquisition Dates of TSX Images } \\
\hline 2010/10/27 & 2011/08/31 & $2013 / 08 / 26$ & 2014/02/07 \\
\hline $2010 / 11 / 18$ & 2011/10/03 & $\begin{array}{c}b d=21 \\
2013 / 09 / 17\end{array}$ & $2014 / 03 / 01$ \\
\hline $2011 / 01 / 23$ & 2011/12/30 & $\begin{array}{c}b d=22 \\
2013 / 09 / 28\end{array}$ & $2014 / 03 / 23$ \\
\hline $2011 / 02 / 14$ & $2012 / 01 / 10$ & $\begin{array}{c}b d=23 \\
2013 / 10 / 20\end{array}$ & $2014 / 05 / 06$ \\
\hline & & $b d=24$ & \\
\hline 2011/03/08 & 2012/02/01 & $2013 / 10 / 31$ & $2014 / 05 / 28$ \\
\hline $2011 / 03 / 30$ & $2012 / 02 / 12$ & $\begin{array}{c}b d=25 \\
2013 / 11 / 22\end{array}$ & $2014 / 06 / 19$ \\
\hline & $-b d=16$ & $-b d=26$ & \\
\hline 2011/06/04 & $2013 / 06 / 21$ & 2013/12/03 & 2014/07/11 \\
\hline $2011 / 06 / 15$ & $\begin{array}{c}b d=17 \\
2013 / 07 / 13\end{array}$ & $\begin{array}{c}b d=27 \\
2013 / 12 / 25\end{array}$ & 2014/08/02 \\
\hline & $h d=18$ & $b d=28$ & \\
\hline 2011/07/18 & $2013 / 07 / 24$ & $2014 / 01 / 05$ & 2014/08/24 \\
\hline 2011/08/ & $2013 / 08 / 1$ & $2014 / 01 / 16$ & 2014/09/04 \\
\hline
\end{tabular}

Table 1. TSX images and break date $(b d)$ setting.

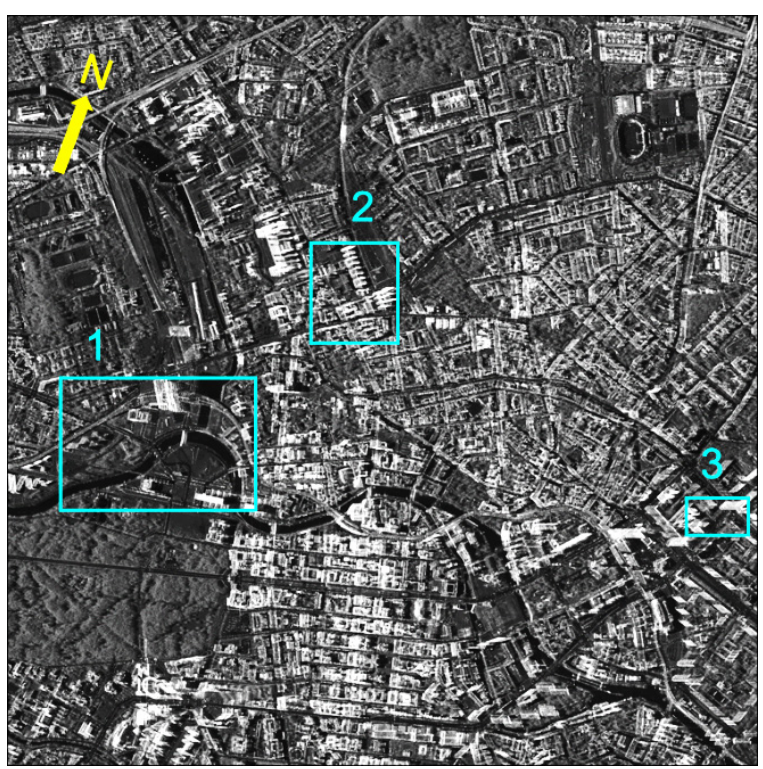

Figure 3. Mean TSX image over study area. Patches 1 to 3 are used for in-depth analysis.

First, the construction events around Berlin Central Station are explored (Figure 5). The office complex of Federal Ministry of the Interior (area 1) had been constructed in the second half of 2013. A series of construction events is present in area 2. The quad-square structures were removed at the early stage. Afterwards, the upper-left hotel had been erected over time by 2013; however, another new hotel and two office buildings cannot be detected because the constructions were still in progress after the last break date. Certain new surface substructures in areas 3, 4, and 7 have been revealed by our method; in contrast, these subtle changes can be hardly seen in the GE images. Another construction event, which is difficult to be recognized from the GE images, is illustrated in area 5 where the bridge was renovated during a couple of early months in 2013 Area 6 displays two new office buildings that have been constructed at different schedules. The right building had been completed earlier, giving rise to a building-shaped pattern formed by clustered EBC points. In contrast, the construction progress on the left building was slower as only sparse EBC points appeared in late 2013 . 


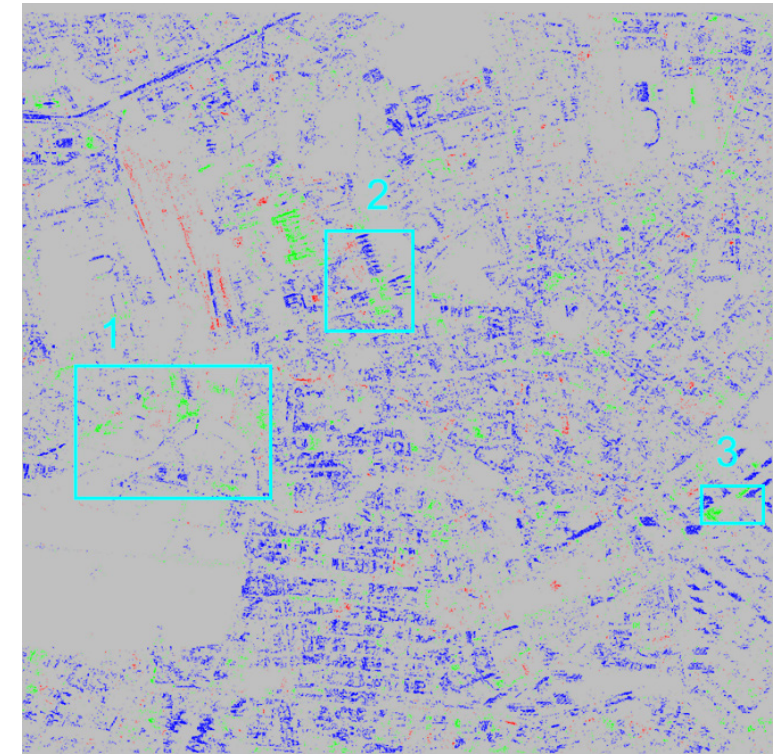

(a)

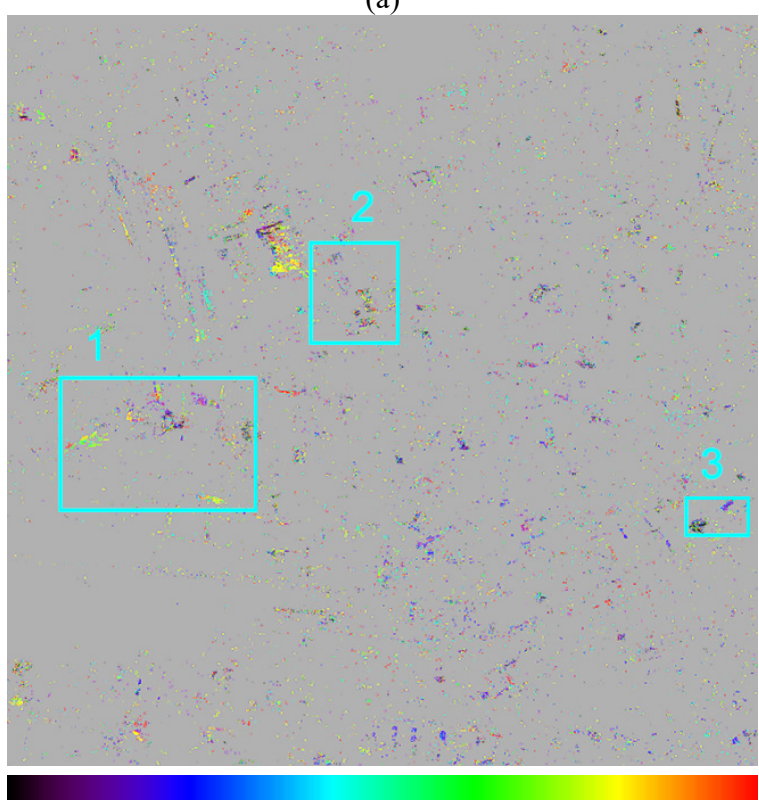

(b)

Figure 4. Spatiotemporal change detection result. Patches 1 to 3 are used for in-depth analysis. (a) Steady, disappearing, and emerging buildings represented by PS (blue, $41277 / \mathrm{km}^{2}$ ), DBC (red, 2200/ $\mathrm{km}^{2}$ ), and $\mathrm{EBC}$ (green, $7180 / \mathrm{km}^{2}$ ) points. (b) Disappearance and emergence dates: black to red, earliest to latest in 2013.

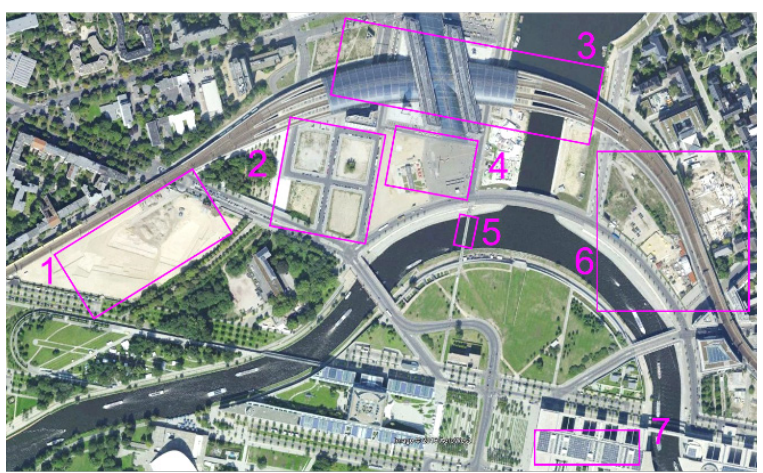

(a)

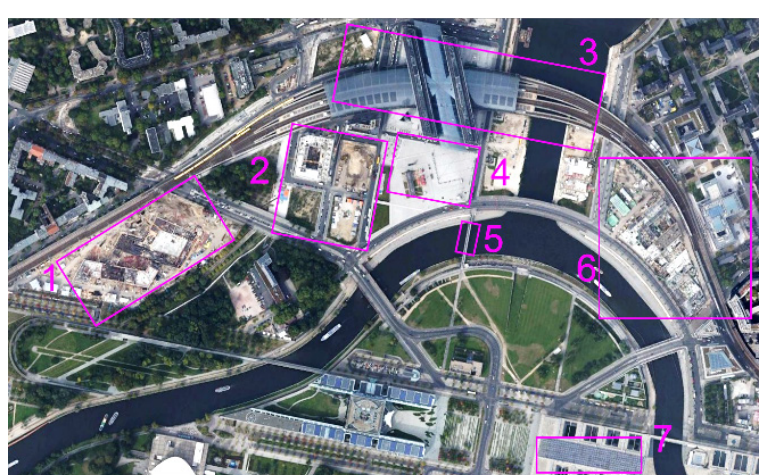

(b)

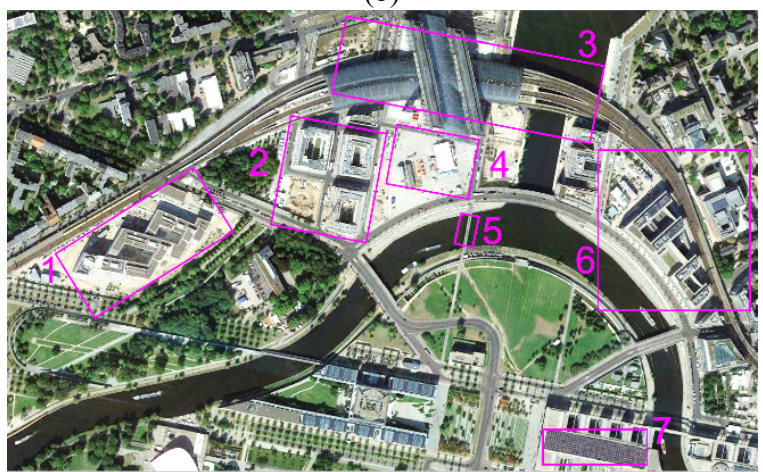

(c)

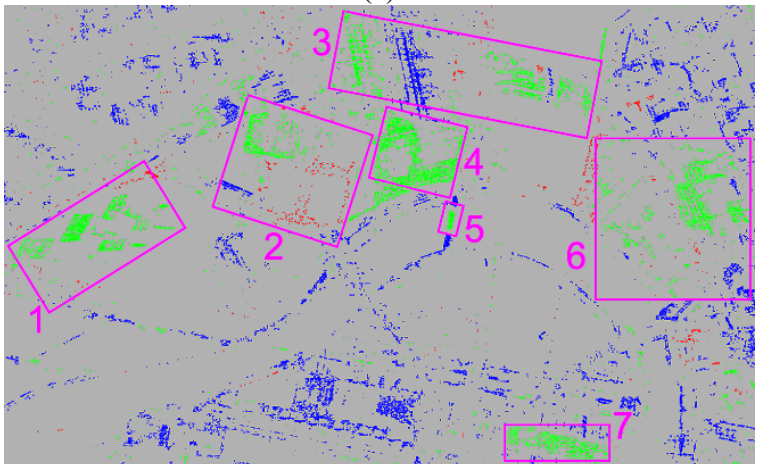

(d)

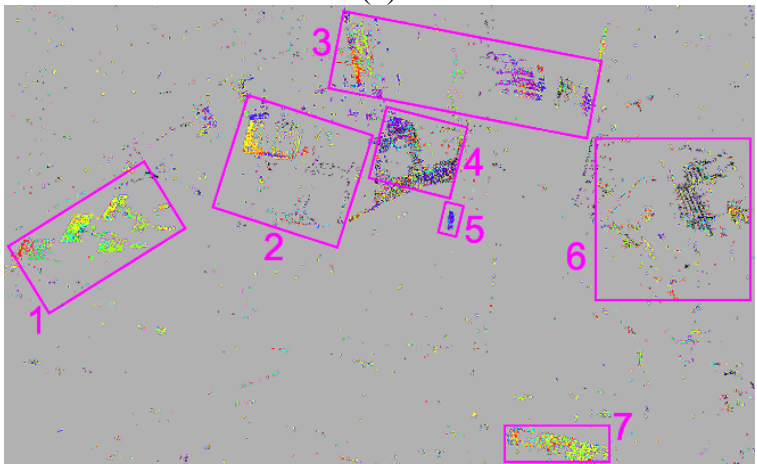

(e)

Figure 5. Construction monitoring in patch 1 (Figure 3) around Berlin Central Station. Areas 1 to 7 are used for in-depth analysis. GE images were acquired on (a) September 12, 2010, (b) May 20, 2012, and (c) September 5, 2014. (d) and (e): spatiotemporal change detection result in patch 1 (Figure 4). 
The second example (Figure 6) is about monitoring a business district, in which building changes are usually frequent and need cost-effective surveillance schemes. In the early 2013, the buildings in areas 1 and 2 were demolished. Meanwhile, the main structures of some new buildings appeared in areas 3 to 7 and the constructions continued to the end of 2013. Since the second half of 2013, certain substructures had been added to the German Railway's office complex in area 8 . These additions seem vague in the GE images but are clearly revealed in our result.

The last example demonstrates construction monitoring of single high-rise buildings (Figure 7). The left building's main structure (area 1) had been erected in early 2013 and the remaining substructures were later complemented over time. In area 2 , certain new storeys were built upon an existing building from low to high level in sequence along the magenta arrow (Figure 7(e)). This example shows that our technique is able to provide detailed spatiotemporal information about construction progress.
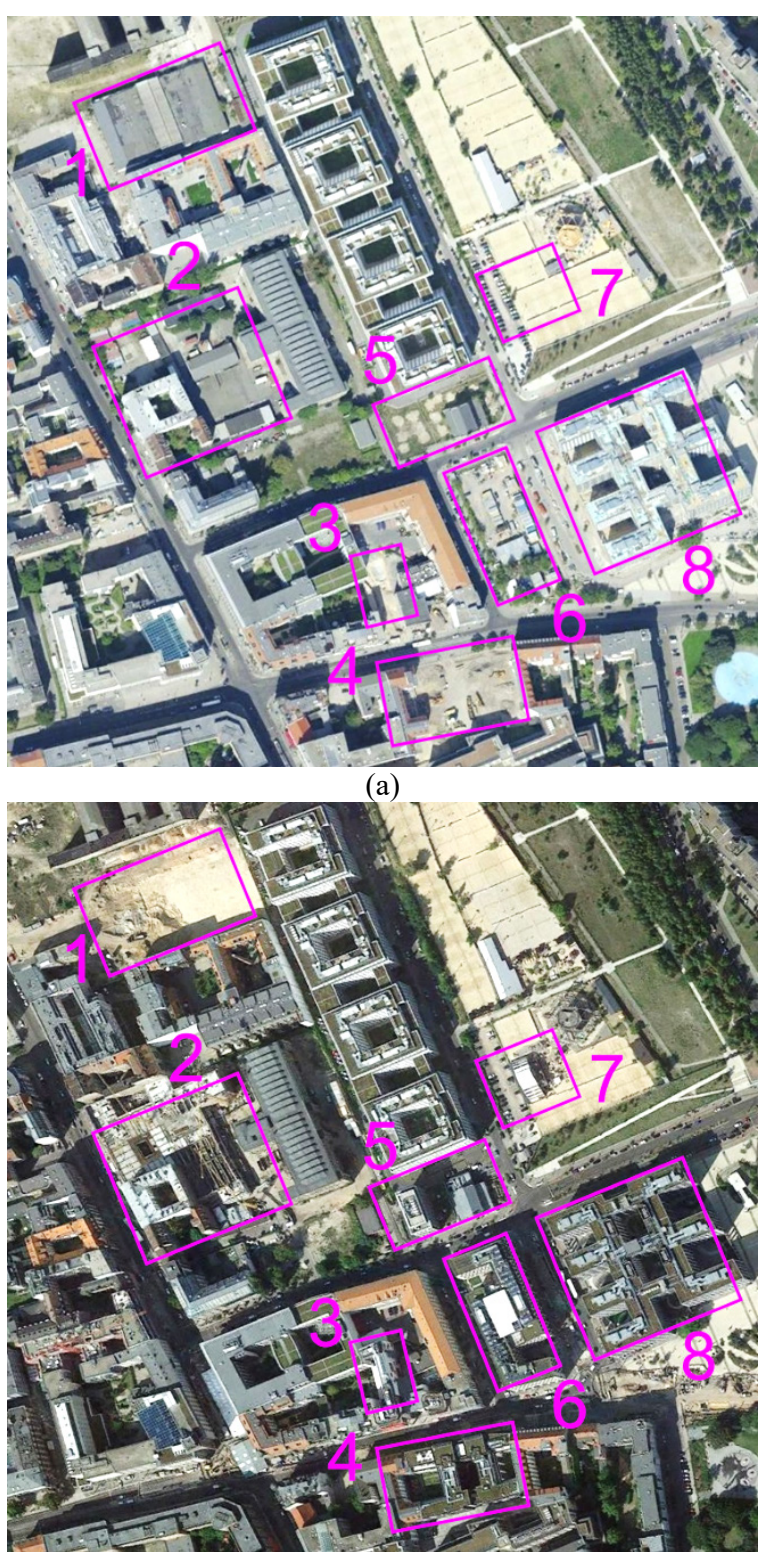

(b)

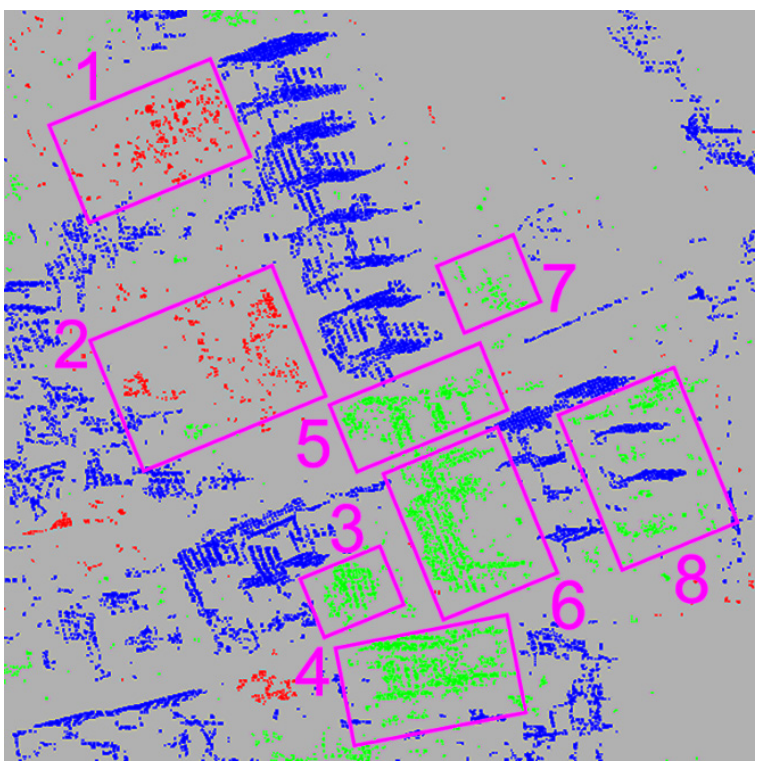

(c)

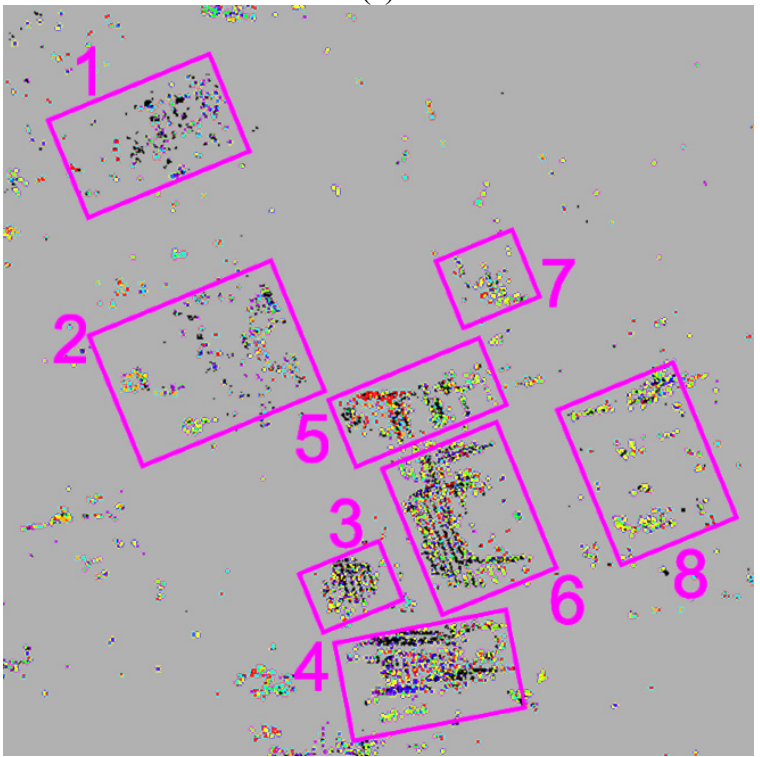

(d)

Figure 6. Construction monitoring of business district in patch 2 (Figure 3). Areas 1 to 8 are used for in-depth analysis. GE images were acquired on (a) September 12, 2010 and (b) September 5, 2014. (c) and (d): spatiotemporal change detection result in patch 2 (Figure 4).

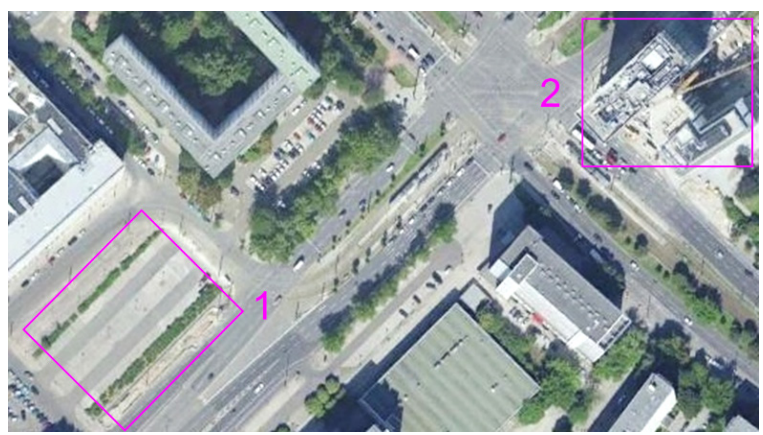

(a) 


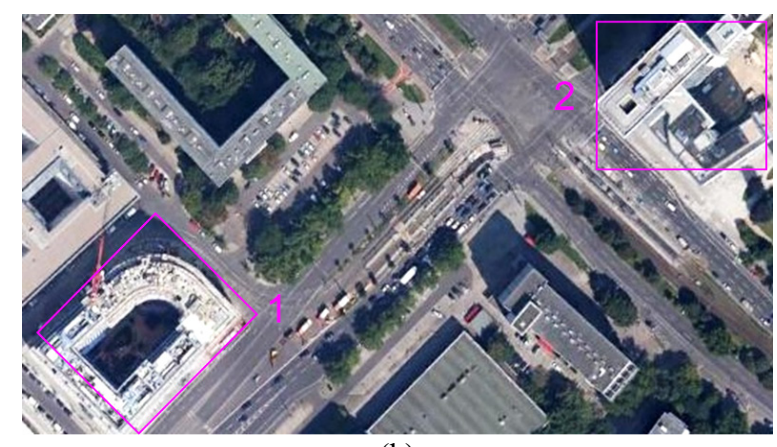

(b)

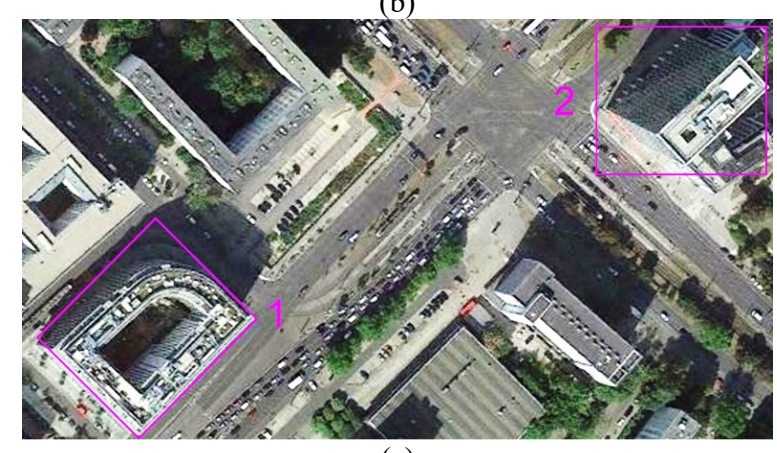

(c)

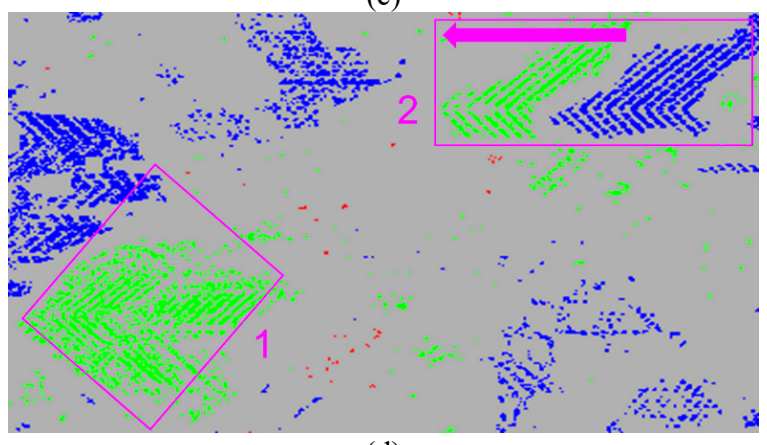

(d)

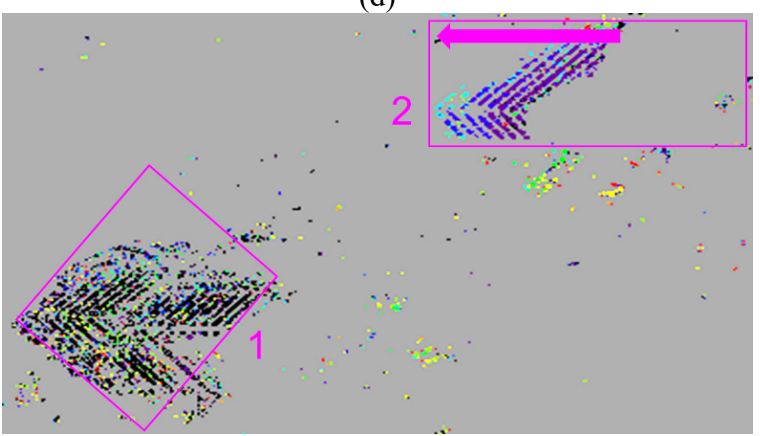

(e)

Figure 7. Construction monitoring of single high-rise buildings in patch 3 (Figure 3). Areas 1 to 2 are used for in-depth analysis. GE images were acquired on (a) September 12, 2010, (b) May 20, 2012, and (c) September 5, 2014. (d) and (e): spatiotemporal change detection result in patch 3 (Figure 4).

\section{OBJECT-BASED ANALYSIS}

Moving from point- to object-based analysis, we select two representative examples for discussion. The first example (Figure 8) explores three new office buildings of Federal Ministry of the Interior. Buildings 2 and 3 were constructed in October 2013. We infer that their construction progresses were almost the same considering that the mean and SD of their emergence dates are close (these values refer to the break dates given in Table I). In more detail, the construction on building 2 (Mean: 23.3) was supposed to be finished a bit earlier than building 3 (Mean: 23.9), if anything. Compared with buildings 2 and 3, building 1 seems to be built one month later in November. In addition, the construction time of building 1 was supposed to be relatively long as its SD of emergence dates is larger than those of other two buildings. The second example (Figure 9) looks into a new highrise building (segment 1 ) and certain new storeys (segment 2 ) on an existing building. The new building was built in July 2013. Many substructures were added to the main structure, which had been completed in the early stage, randomly in space and time. This activity is credited to the temporal variation, i.e., SD of 2.7, during the construction. The new storeys were constructed roughly between July and August in 2013. The fact that these storeys were layered gradually from bottom to top gives rise to the temporal variation indicated by SD of 2.1. In summary, object-based analysis gives assistance in understanding of single construction events in an efficient way. If need be, detailed investigations on events of interest can resort to point-based analysis.

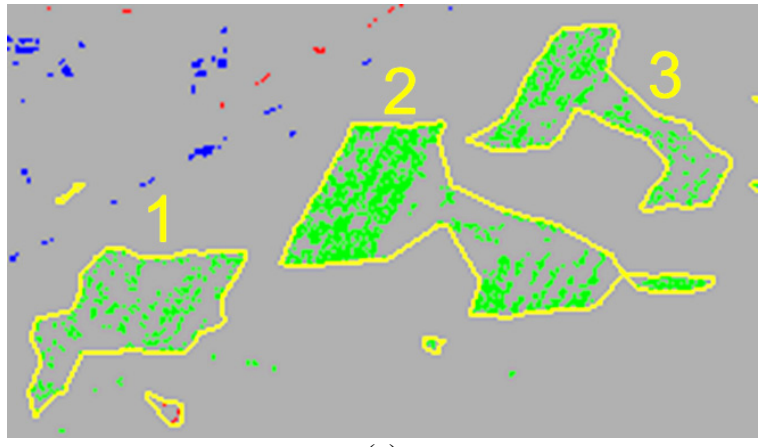

(a)

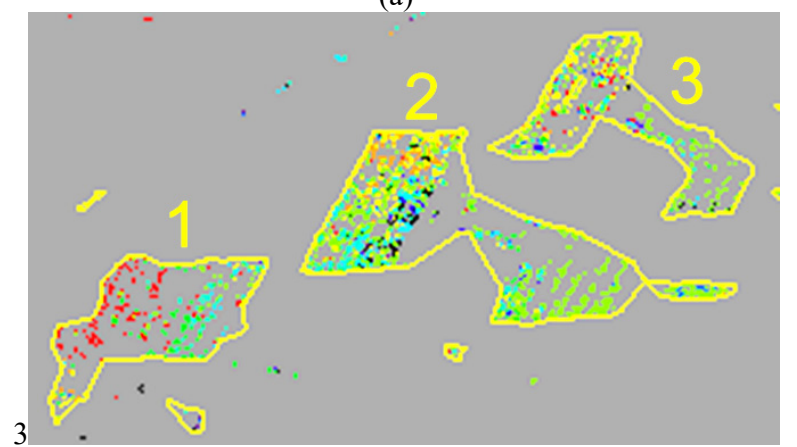

(b)

Figure 8. Spatiotemporal changes within area 1 in patch 1 (Figure 5). (a) EBC points are clustered as segments 1, 2, and 3 that correspond to three new office buildings of Federal Ministry of the Interior. (b) Mean and SD of emergence dates (unit: $b d$ defined in Table 1). Segment 1: Mean, 25.1; SD, 3.1. Segment 2: Mean, 23.3; SD, 2.2. Segment 3: Mean, 23.9; SD, 2.0.

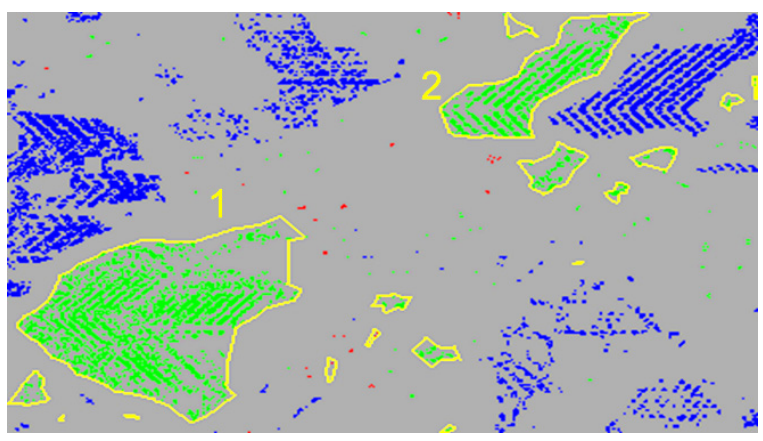

(a) 


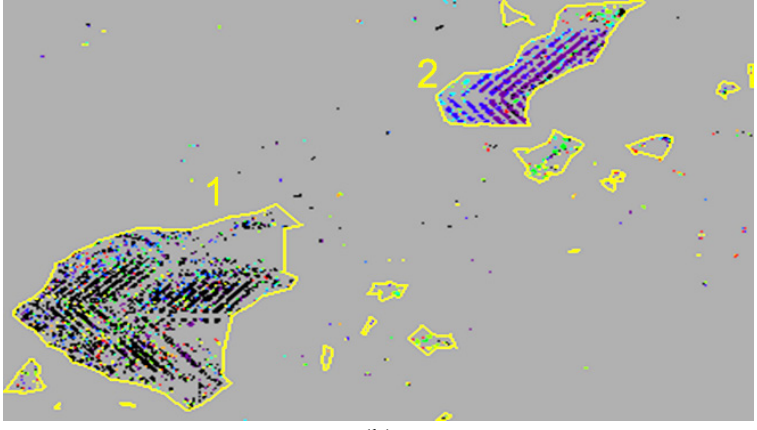

(b)

Figure 9. Spatiotemporal changes in patch 3 (Figure 7). (a) EBC points are clustered as segment 1 (new high-rise building) and segment 2 (new storeys). (b) Mean and SD of emergence dates (unit: $b d$ defined in Table 1). Segment 1: Mean, 18.4; SD, 2.7. Segment 2: Mean, 19.1; SD, 2.1.

\section{CONCLUSIONS}

Previously, we proposed a 4D change detection technique to detect spatial changes (3D) and occurrence times (1D). In this study, we apply our further developed method to enable construction monitoring in more detail. The aims of the three case studies are to monitor construction progress, business districts, and single buildings. As to construction monitoring, three main construction types, i.e., demolition, erection, and renovation, can be distinctly recognized along with change times that substructures are added or removed. Such spatiotemporal change information is able to be derived from a business district characterized by frequent and intensive building changes. In more detail, construction progress of single buildings can be obtained with spatiotemporal interpretations. One typical example in our test reveals that several storeys were built upon an existing high-rise building layer by layer. We demonstrate that object-based analysis is helpful for efficient understanding of single construction events. Then, point-based analysis targeting substructures can be conducted for more detailed investigation if necessary. In summary, our method is applicable to monitor building construction with detailed and comprehensive spatiotemporal information. For future works, we will work on a study area in Stuttgart, Germany, where many constructions, e.g., the main station and traffic lines, started since 2010 and require cost-effective monitoring approaches for municipal administration.

\section{REFERENCES}

Ansari, H., Adam, N. and Brcic, R., 2014. Amplitude time series analysis in detection of persistent and temporal coherent scatterers. Proceedings of IEEE IGARSS, Quebec, Canada, pp. 2213-2216.

Berardino, P., Fornaro, G., Lanari, R. and Sansosti, E., 2002. A new algorithm for surface deformation monitoring based on small baseline differential SAR interferogram. IEEE Transactions on Geoscience and Remote Sensing, 40(11), pp. 2375-2382.

Brcic, R. and Adam, N., 2013. Detecting changes in persistent scatterers. Proceedings of IEEE IGARSS, Melbourne, Australia, pp. 117-120.

Crosetto, M., Monserrat, O., Cuevas-González, M., Devanthéry, N. and Crippa, B., 2016. Persistent scatterer interferometry: a review. ISPRS Journal of Photogrammetry and Remote Sensing, 115, pp. 78-89.
Edelsbrunner, H., Kirkpatrick, D. and Seidel, R., 1983. On the shape of a set of points in the plane. IEEE Transactions on Information Theory, 29(4), pp. 551-559.

Ester, M., Kriegel, H., Sander, J. and Xu, X., 1996. A densitybased algorithm for discovering clusters in large spatial databases with noise. Proceedings of 2nd International Conference on Knowledge Discovery and Data Mining, Portland, America, pp. 226-231.

Ferretti, A., Prati, C. and Rocca, F., 2000. Nonlinear subsidence rate estimation using permanent scatterers in differential SAR interferometry. IEEE Transactions on Geoscience and Remote Sensing, 38(5), pp. 2202-2212.

Ferretti, A., Prati, C. and Rocca, F., 2001. Permanent scatterers in SAR interferometry. IEEE Transactions on Geoscience and Remote Sensing, 39(1), pp. 8-20.

Ferretti, A., Colesanti, C., Perissin, D., Prati, C. and Rocca, F., 2003. Evaluating the effect of the observation time on the distribution of SAR permanent scatterers. Proceedings of FRINGE, Frascati, Italy.

Ferretti, A., Fumagalli, A., Novali, A., Prati, C., Rocca, F. and Rucci, A., 2011. A new algorithm for processing interferometric data-stacks: SqueeSAR. IEEE Transactions on Geoscience and Remote Sensing, 49(9), pp. 3460-3470.

Gamba, P., 2013. Human settlements: a global challenge for EO data processing and interpretation. Proceedings of the IEEE, 101(3), pp. 570-581.

Hooper, A., Zebker, H., Segall, P. and Kampes, B., 2004. A new method for measuring deformation on volcanoes and other natural terrains using InSAR persistent scatterers. Geophysical Research Letters, 31(23), pp. 1-5.

Kampes, B. M., 2006. Radar Interferometry: Persistent Scatterer Technique. Springer.

Lanari, R., Mora, O., Manunta, M., Mallorquí, J. J., Berardino, P. and Sansosti, E., 2004. A small baseline approach for investigating deformations on full resolution differential SAR interferograms. IEEE Transactions on Geoscience and Remote Sensing, 42(7), pp. 1377-1386.

Marin, C., Bovolo, F. and Bruzzone, L., 2015. Building change detection in multitemporal very high resolution SAR images. IEEE Transactions on Geoscience and Remote Sensing, 53(5), pp. 2664-2682.

Mittermayer, J., Wollstadt, S., Prats-Iraola, P. and Scheiber, R., 2014. The TerraSAR-X staring spotlight mode concept. IEEE Transactions on Geoscience and Remote Sensing, 52(6), pp. 3695-3706.

Novali, F., Basilico, M., Ferretti, A., Prati, C. and Rocca, F., 2004. Advances in permanent scatterer analysis: semi and temporary PS. Proceedings of EUSAR, Berlin, Germany.

Yang, C. H., Kenduiywo, B. K., and Soergel, U., 2016. 4D Change Detection Based on Persistent Scatterer Interferometry. Proceedings of ICPR\&PRRS, Cancun, Mexico. 


\section{APPENDIX}

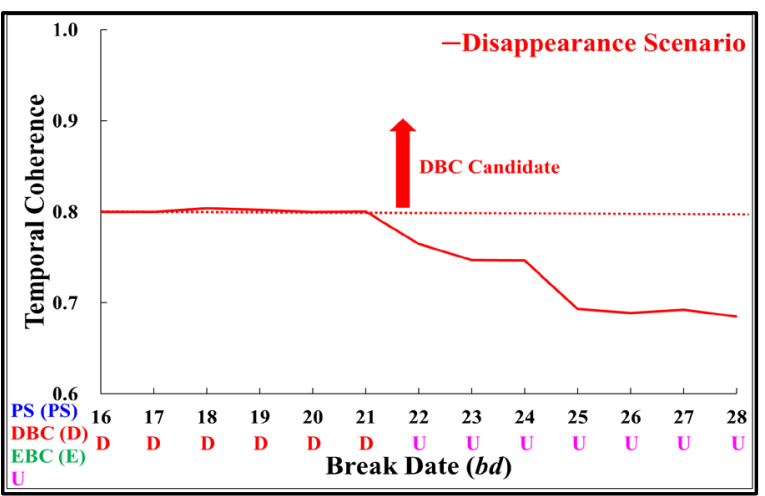

(a)

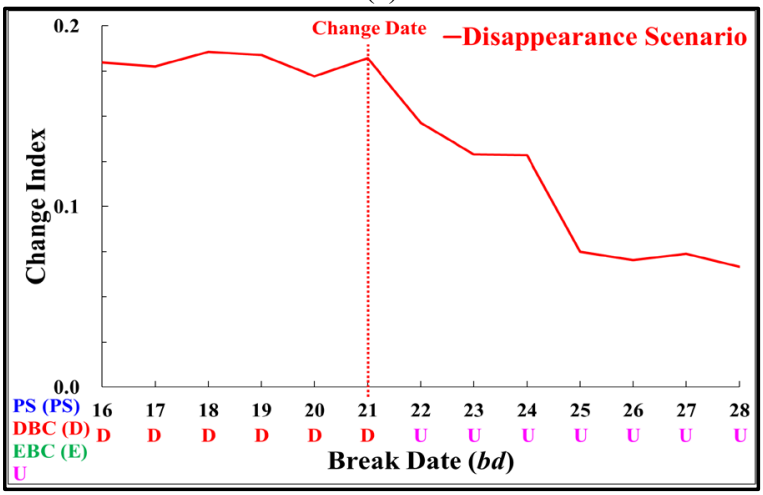

(b)

Figure A.1. Example of DBC point's (a) temporal coherence sequence and (b) chance index sequence along with initial point labels.

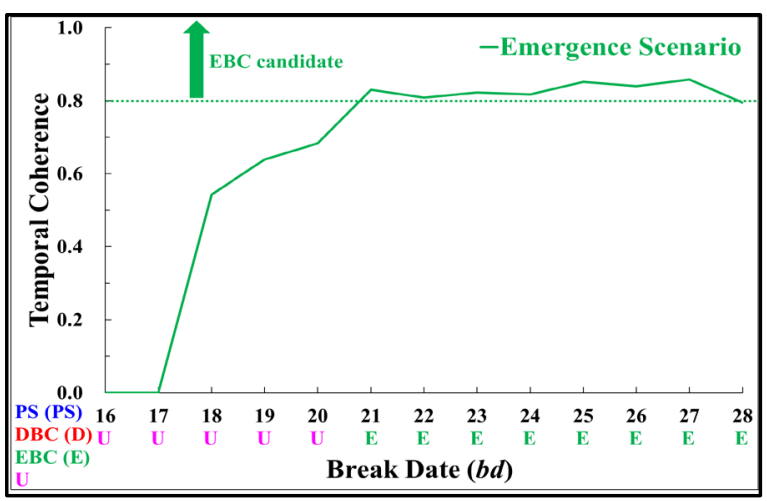

(a)

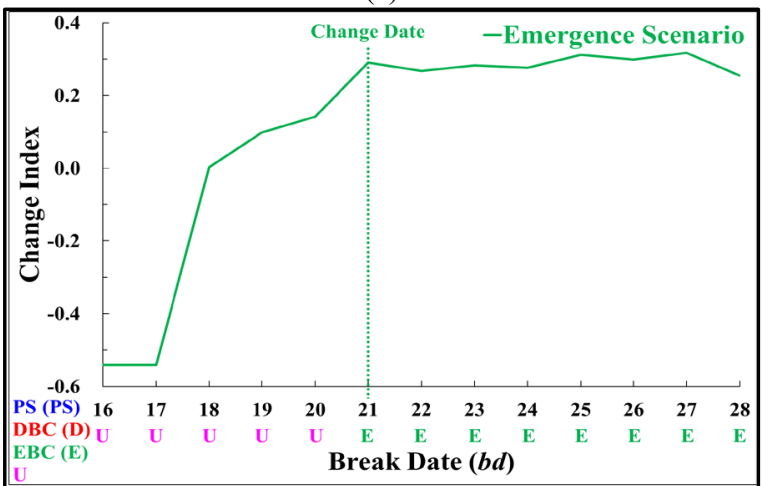

(b)

Figure A.2. Example of EBC point's (a) temporal coherence sequence and (b) chance index sequence along with initial point labels. 